\title{
Radiobiological foundation of crew radiation risk for Mars mission to the problem of the space flight safety
}

\author{
Aleksandr Shafirkin ${ }^{1}$, Yury Grigoriev ${ }^{2}$ \\ ${ }^{1}$ Lab. of the Space Flights Radiation Safety of State Research Center - Institute for biomedical problems Russian Academy of Sciences, \\ Moscow, Russia \\ ${ }^{2}$ Lab. of the Electromagnetic Safety of State Research Center - Federal medical biophysical Burnazian centre, Moscow, Russia
}

Email address:

a.v.shafirkin@mail.ru (A. Shafirkin), profgrig@gmail.com (Yu. Grigoriev)

\section{To cite this article:}

Aleksandr Shafirkin, Yury Grigoriev. Radiobiological Foundation of Crew Radiation Risk for Mars Mission to the Problem of the Space Flight Safety. American Journal of Life Sciences. Special Issue: Space Flight Factors: From Cell to Body. Vol. 3, No. 1-2, 2015 , pp. $32-42$. doi: 10.11648/j.ajls.s.2015030102.16

\begin{abstract}
This paper presents results of radiobiological experiments, a new concept of radiation hazard on the basis of a generalized dosimetric function, which are foundation of crew radiation risk for Mars mission. The results of 14-year biological experiment on comprehensive clinico-physiological study of $250 \mathrm{dogs}$ exposure to gamma-radiation ${ }^{60} \mathrm{Co}$ during 3 and 6 years 22 hours per day, modeling the impact of space radiation on the crew of a space shuttle flight to Mars are presented also. In this experiment dose and dose rate varied to simulate galactic cosmic ray (GCR) dose and dose from stochastic irradiation caused by solar cosmic rays (SCR). It details observations made on the animals throughout the course of the dogs' lives, both during and eight years after radiation exposure. It includes data on dose dependence, threshold levels of radiation causing adverse health effects, as well as on the nature of radiation reactions as they develop in different organs and body systems chronically exposed to larger doses. This multi-year experiment was conducted by scientists of the State Research Center Institute of Biomedical Problems (Russian Academy of Sciences) with the active participation of the Institute of Biophysics (now Burnasyan Federal Medical Biophysical Center of Federal Medical Biological Agency). Based on results obtained in this study and in experiments realized with big amount of small laboratory animals that were exposed to a wide dose and dose rate range, using other published data, mathematical models were developed, e.g. a model of radiation damage forming as dependent on time with taking into account recovery processes, and model of radiation mortality rate of mammals. Based on these models and analysis of radiation environment behind various shielding on the route to Mars, crew radiation risk was calculated for space missions of various duration. Total radiation risk values for cosmonauts lifetime after the missions were also estimated together with expected life span reduction.
\end{abstract}

Keywords: Effects of Chronic and Acute Irradiation, Mathematical Models of Radiation Damage, Life Span Reduction, Radiation Risk for Cosmonauts Lifetime

\section{Introduction}

Dose-time dependencies for acute and delayed effects after the standard radiation impact on the Earth have been studied rather well. The standard radiation impact is regarded as an acute uniform exposure from sources of ionizing radiation, characterized by the quality factor of 1 , (X-rays or gamma radiation). These data can be also used to estimate the risk of the lowering of human working capacity resulted from acute and continual irradiation in different doses, as well as risk of distant unfavorable effects. Unlike radiation impact character on the Earth, defined mainly by radiation with a low value of
Linear Energy Transfer (LET), and being uniform in most cases, the exposure to radiation under conditions of a space flight is characterized by a complicated radiation composition and energetic spectrum ranging from several $\mathrm{keV}$ to hundreds of $\mathrm{GeV}$, separate components of which vary significantly by their biological efficiency. In addition it is characterized by non-uniform dose distributions both by a body depth and time.

To determine the hazard of the cosmic radiation impact and the probability of the lowering of a spacecraft crew 
working capacity during a flight and possible distant postflight unfavorable effects (an increase in death risk during lifetime and the shortening of life expectancy) it is necessary to put into use a specific dosimetric functional which allows a complicated nature radiation impact in space (chronic irradiation from GCR and stochastic distribution acute doses from SCR in period maximum solar activity (SA) to be reduced to the conditions of the standard radiation impact. Such a functional called the generalized dose has been designed.

\section{Conception of Cosmonaut's Radiation Hazard Estimation During Long Term Space Missions on Basis of Generalized Dosimetric Functional}

In result presented numerous experimental investigation to determine the hazard of the cosmic radiation exposure and calculations total radiation risk was developed a new special dosimetric function - the "generalized dose", $\mathrm{H}$ [1-3].

That dosimetric function has allowed for the complex composition and spectrum of cosmic radiations, for the spatial and temporal inhomogeneity of the radiation impact, and for the occurrences of the non-exposure factors that have a great influence on the radiation-induced response of an organism. It is necessary also to stress the following thing. The acute radiation effects during a manned space flight and dangerous long term consequences are associated with forming pathological changes in essentially different critical organs and tissues of an organism distinguished by the time for the development of damages, their localization and the rate of recovery processes. That is why values of generalized dose should be calculated separately, based on the these set of coefficients, respectively, for nearly the in-flight effect $\mathrm{H}_{\mathrm{N}}$ and later long term consequences $\mathrm{H}_{\mathrm{L}}$ :

$$
\begin{aligned}
& \mathrm{H}_{\mathrm{N}}=\left(\sum_{\mathrm{i}=1}^{\mathrm{n}} \overline{\mathrm{Di}}[\mathrm{Gy}] * \mathrm{QF}_{\mathrm{Ni}} * \mathrm{TF}_{\mathrm{Ni}} * \mathrm{SF}_{\mathrm{Ni}}\right) * \mathrm{MF}_{\mathrm{N}} \\
& \mathrm{H}_{\mathrm{L}}=\left(\sum_{\mathrm{i}=1}^{\mathrm{n}} \overline{\mathrm{Di}}[\mathrm{Gy}] * \mathrm{QF}_{\mathrm{Li}} * \mathrm{TF}_{\mathrm{Li}} * \mathrm{SF}_{\mathrm{Li}}\right) * \mathrm{MF}_{\mathrm{L}}
\end{aligned}
$$

where Di is a mean-tissue absorbed dose; $Q F_{N i}$ and $Q F_{L i}$ are quality factors of ionizing radiation, respectively, for in-flight and late radiobiological effects from the $i$-th source of irradiation; $T F_{N i}$ and $T F_{L i}$ are coefficients of temporal nonuniformity of radiation exposure accounting for the influence on the effects of a dose rate and the pattern of temporal dose distribution; $S F_{N i}$ and $S F_{L i}$ are coefficients of spatial nonuniformity of irradiation, considering a dose distribution pattern inside a human body and converting the effects of nonuniform irradiation to the effects of uniform radiation exposure, respectively, for the in-flight and late effects from the $i$-th source of radiation exposure in the space; $M F_{N}$ and $M F_{L}$ are modification coefficients of radiation response of an organism through the additional effects caused by nonradiation factors during a flight. In term of the generalized dose it is possible to assess radiation risk in flight and lifetime total radiation risk of cosmonauts and present assessments of possible shortening of life expectancy [1-3].

The radiobiology laboratories of Institute of Biomedical Problems made multi-purpose studies of cosmic ray effects. The 35-year-long researches on the proton and ion accelerators have established the dependence of the of relative biological efficiency (RBE) factors on LET. The results of the researches have made it possible to find the mean quality factors (QF) for different cosmic radiation sources as applied to the estimation of the nearly and late radiobiological effects [3-5].

The experiments with chronic and repeated irradiations of animals using the gamma-irradiation facilities have found the influence of the dose, the dose rate, and the character of temporal dose distribution on the radiobiological effects. In order to take into account the dynamics of irradiation during a space flight a model of the effective residual dose Def was used, which was suggested in works [6-7] for the first time and improved then by us $[2,3,8]$. As a result, a new mathematical model of the effective residual dose $D_{\text {ef }}$ has been constructed for forming radiation damages under longterm and repeated acute exposures, that allows more just to take in consideration the recovery processes in cells, tissues, and organism as a whole.

It was represented as:

$$
\mathrm{dD}_{\text {ef }} / \mathrm{dt}=\mathrm{K}(\gamma) \gamma(\mathrm{t}), \text { for } \mathrm{t} \leq \tau
$$

$$
\mathrm{dD}_{\mathrm{ef}} / \mathrm{dt}=\mathrm{K}(\gamma) \gamma(\mathrm{t})-\beta_{0}\left(\mathrm{e}^{-[\lambda 0+\lambda \mathrm{R}(\gamma, \mathrm{t})] \mathrm{t}}\right) \mathrm{D}_{\mathrm{ef}}(\mathrm{t}-\tau) \text { for } \mathrm{t}>\tau \text {, }
$$

where $D_{\text {ef }}$ - effective residual dose, $c G y ; \gamma$-dose rate; $K(\gamma)$ - coefficient reduction of radiation impact efficiently connected with fast recovery processes on cell level; $\beta_{0}$ coefficient, determing of a velocity recovery processes for young animals on organism level as a whole; $\lambda_{0}$ - index, related with processes of aging; $\lambda_{\mathrm{R}}(\gamma, \mathrm{t})$ - index of velocity recovery processes reduction, determing with total dose irradiation D, cGy.

$$
\begin{gathered}
\lambda_{\mathrm{R}}(\gamma, \mathrm{t}) \approx \beta_{0} \exp (-0,0009 \mathrm{D}) ; \mathrm{K}(\gamma)=\left(\gamma / \gamma_{\mathrm{B}}\right)^{0,18}(\text { Fig. } 1), \text { where } \\
\gamma_{\mathrm{B}}=40 \mathrm{~Gy} / \text { day }
\end{gathered}
$$

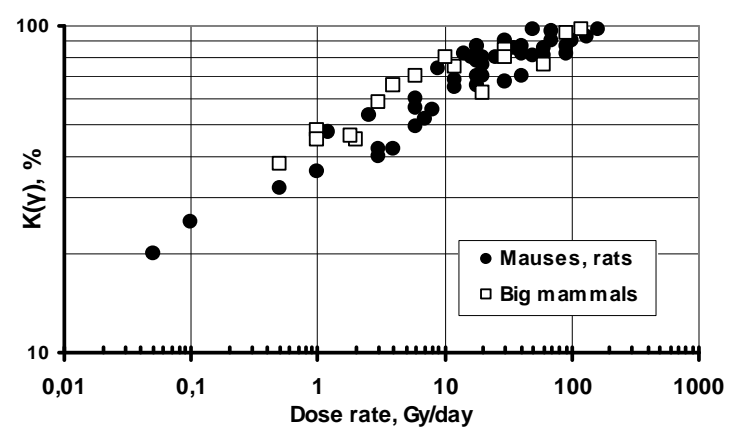

Figure 1. Efficiently reaction of blood form system (\%) from dose rate, connected with quick recovery processes in cell level (experiments on big mammals and small laboratory animals 
This model has made it possible to satisfactorily describe the diverse radiobiological experiments that can be found in literature. The model was also used to estimate the time factor (TF) for different cosmic radiation sources as applied to the nearly and late radiobiological effects.

The researches into non-uniform irradiation of the animals with different local shieldings of separate areas of their bodies have made it possible to construct the mathematical model of the equal dose equivalent $G[D(r)]$ for reducing the non-uniform exposure effects to the standard uniform exposure. The model has underlain the techniques for determining the spatial factor (SF) of spatial non-uniformity of the exposure to cosmic radiation $[1,9]$.

Diverse researches into the combined impact of the exposure and non-exposure factors (acceleration, vibration, hypoxia, hyperthermia, exercise load, electromagnetic radiations, etc.) have been carried out. The modification factors of the radiation response of an organism have been evaluated in dose units. The modification factors (MF) have been evaluated within range $0.85-1.30$ by analyzing the research results obtained at out Institute and published elsewhere [1-3, 10, 11].

\section{Special Experiment for Determination Radiation Dangerous Long Term Space Mission to Mars and Assessment of Crew Radiation Risk}

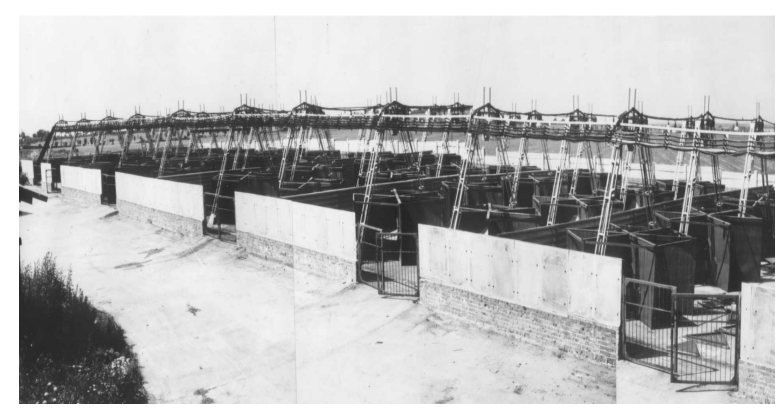

Figure 2. The area for exposure dogs

In terms of the radiation hazard problem, the following experimental research is of particular interest. The prestarting procedure for a many-year experiment on a large group of dogs was initiated as long ago as 1964. Already in 1966 year was begin chronic experiment in which 250 dogs were exposed to gamma-radiation during 3 and 6 years on 22 hours per day. In this experiment dose and dose rate varied to simulate galactic cosmic ray (GCR) dose and dose from stochastic irradiation caused by solar cosmic rays (SCR) during long term space mission to Mars. A mean-tissue equivalent dose rate in 1;2 and 3-th groups of animals simulate GCR dose was consisted 21, 62 and $125 \mathrm{cSv} /$ year respectively. The experimental area for exposure dogs represent on figure 2 . The animals were irradiated in a special devices whose main component was a collimator containing a ${ }^{60} \mathrm{Co}$ gamma sources. The collimator was place above a cage where six dogs were kept simultaneously (Figure 3).

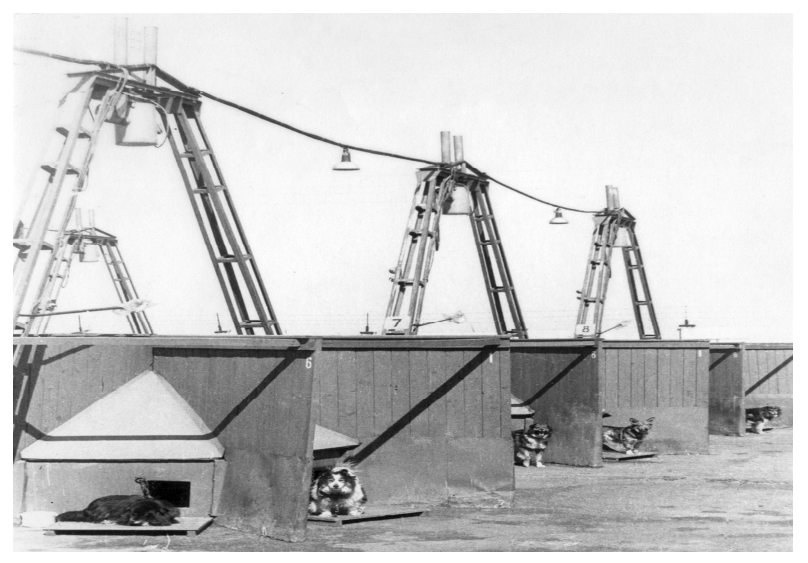

Figure 3. Course of gamma-rays and cage with experimental dogs

In addition to chronic irradiation with dose rate 62 cSv/year simulating GCR effect, the animals in 4-th and 5-th groups were also exposed to acute irradiation through each 4 month in mean-tissue equivalent doses 42 and $8 \mathrm{cSv}$ simulating the impact of solar cosmic rays. This exposure was to simulate dosage and time effects of powerful solar flares, for instance the flares of 23 February 1956, 10 May 1959 and 13 February 1960. The irradiation procedure and the equivalent doses are given in Table 1.

Table 1. Irradiation procedure and equivalent dosage in experiment

\begin{tabular}{|c|c|c|c|c|c|}
\hline \multirow{2}{*}{ Group N } & \multicolumn{5}{|c|}{ Equivalent mid-tissue dose, $\mathrm{cSv}$} \\
\hline & Chronic exposure per year & Acute exposure & Total dose per year & Total dose per 3 year & Total dose per 6 year \\
\hline 1 & 21 & - & 21 & 63 & 126 \\
\hline 2 & 62 & - & 62 & 186 & 372 \\
\hline 3 & 125 & - & 125 & 375 & 750 \\
\hline 4 & 62 & 4288 & 120 & 360 & 720 \\
\hline 5 & 62 & 424242 & 188 & 564 & 1128 \\
\hline $6(\mathrm{~K})$ & 0 & 0 & 0 & 0 & 0 \\
\hline
\end{tabular}

The animals were examined throughout their lives in each two months during the chronic exposure, and in each four months after the exposure (the examinations were more frequent after acute exposures). During and after the manyyear exposure, the dogs were examined to check their general clinical state, to study their metabolism by biochemical blood and tissue analyses, to study their hemopoetic tissue morphologically (included analysis of cytokinesis, cell production and velocity of evacuation cell from blood flow), to determine the state of their higher nervous activity and cardiovascular system in normal conditions and under functional loads. The reproductive function of the dogs was 
studied basing on the state of their spermatogenesis and ovogenesis and on the viability and physical growth and development of pups. The various organs and tissues were studied histologically, and some other specialized tests were carried out. There is particular base of date about this experiment $[2,8]$.

\subsection{Condition of Hemopoiesis}

The dogs of groups 2-5 exposed to dose rates of 0.17 cSv/day and higher had safely shown a decreased leukocyte number and an increased reticulocyte level in their blood. The changes were most clearly seen in the animals of the fourth and fifth groups. A significant decrease in the bone marrow reserve of mature granulocytes was found by the pyrogenal test as early as 6 months. Concentration of granulocytes was decreased in 2 and 5 groups to 60 and $20 \%$ accordingly. The changes in the leukoblastic system were apparently of the inhibitory type. By the end of the first year of the experiment, the dogs had a somewhat suppressed white germ of hemopoiesis and a decreased leucoerythroblastic ratio.

During first two years the leukocyte count in the peripheral blood of the dogs was decreased to $75-85 \%$ in comparison with the control (Figure 4).

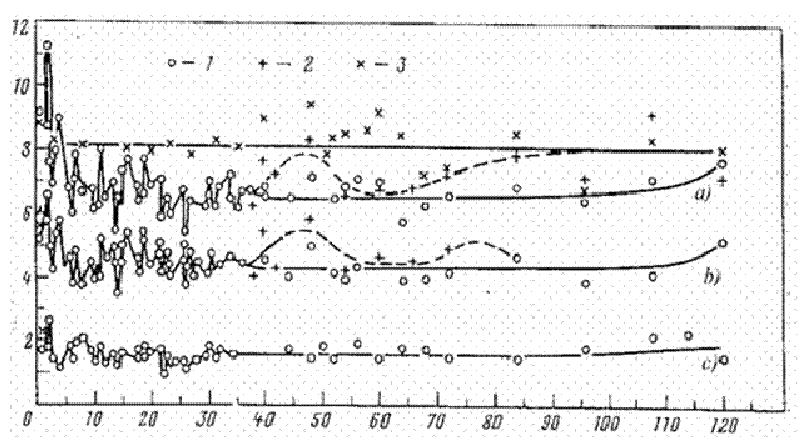

Figure 4. Concentration leukocytes (a), neutrophils (b) and lymphocytes (c) in the peripheral blood dogs in different time from irradiation start 5-th group exposed 6 and 3 year (1) and (2) respectively; (3)-data of control group. Abscissa: time since the start of the exposure, (months); ordinate: blood cells per $1 \mathrm{~mm}^{3} \times 10^{3}$

By the end of the second year of exposure the decrease of the peripheral leukocyte count was followed by a reduction in the circulating and capillary granulocyte pools and a decrease in the half period of granulocyte circulation in the peripheral blood (to $76 \%$ compared with the control). The decrease was evidently associated also with cut of the concentration young fissionable cells in the bone marrow (myeloblasts, promyelocytes and myelocytes). To this period the dogs of groups 3-5 had stabilized the cell composition of their peripheral blood and bone marrow, notwithstanding a decreasing number of the young fissionable cells in their bone marrow (see Fig. 4). This is due to a marked enhancement of the proliferative activity of fissionable cells and to the shorter (by a factor of about 2.5) cellular cycle of myeloblasts, promyelocytes, and particularly myelocytes.

Erythropoietic change different from those peculiar to leukopoiesis. In primary period of exposure the peripheral count of erythrocytes and reticulocytes different insignificantly in comparison with the reference level, remaining within the physiological limits. The number of erythroid elements in bone marrow increased gradually. As early as by the end of the second year of the exposure, the dogs had shown a significant activity of erythropoiesis. Cytological study of bone marrow gave evidence that an increase in erythroid cells was due to a significant increase of the relative number of polychromatophil erythroblasts (beginning from the 16th month up to $130 \%$ compared with the control). At the same time the number of cells of early generation (proerythroblasts and basophil erythroblasts) declined slightly.

By the end of the second year exposure the cell kinetics data indicated a more rapid destruction of erythrocytes, a decreased half-period of their evacuation, $\mathrm{T}_{1 / 2}$ from blood flow, and an increased production of erythrocytes by bone marrow. This did not however bring to an anemic syndrome. The process of accelerated death of erythrocytes was compensated for by an increase in bone marrow production of erythroid elements and mature erythrocytes. The mitotic activity of the polychromatophil erythroblasts had increased to $150 \%$. It was deposed about a formation compensatory reaction to maintain the erythrocyte count at the level required for the basic respiratory function of the blood. The above-presented data show that, despite a certain stabilization concentration cells in the peripheral blood and bone marrow after two years of the exposure date of the cells kinetic were essentially changed. The hemopoiesis process much more intensively, thus indicating a sufficiently high stress of the regulatory systems of organism $[2,8]$.

\subsection{Spermatogenesis and Condition of the Progeny}

Among the radiation-sensitive tissues, the spermatogenous epithelium showed the most pronounced changes. Group 4 showed a clear repeated reaction to the acute $42 \mathrm{cSv}$ dose irradiations (Fig.5). After every acute irradiation in this dose amount of spermatozoa reduced in 8 times and then recovered through 10 months by the beginning of next exposure to that dose per a year. The mean concentration was kept at $15-20 \%$ of the reference level. By the end of the sixth year of the exposure, the dogs of groups 3 and 4 had shown a decrease (by a factor of above 2) in the mass of their sex glands that was accompanied by disturbances of their spermatogenous cycle. The disturbances were evidenced in changing the quantitative relationship among of the different degree of differentiation cells, as well as in decreasing the absolute number of cells, especially during the late stages of their evolution (Fig 5).

In the group $5 \mathrm{dogs}$, concentration of the spermatozoa in their testicles had not been recovered by the moment of a nest exposure to the $42 \mathrm{cSv}$ dose. The damage was cumulatively enhanced in the system of the spermatogenous epithelium during the exposure. The spermatozoa concentration had decreased by factor 30 by the end of the third year and by factor 300 and even higher by the end of 
the sixth year. The decrease in the concentration of spermatozoa was accompanied by a marked fall of their mobility and by an increase in the relative number of the atypical (less viable) forms of cells. Histomorphological investigations confirmed the occurrence of testicular damage. They involved depletion of the testicular tubules and hardening and collagenization of the connective tissue of their walls.

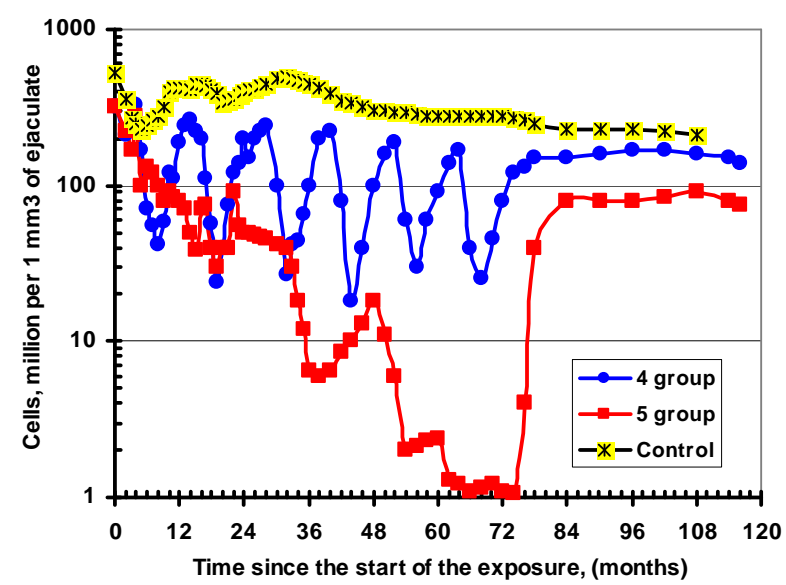

Figure 5. Time-course variations of spermatozoa in the ejaculate of Group 4 and 5 dogs exposed to combined (chronic and acute) irradiation. Arrows show acute exposures. Abscissa: time since the start of the exposure, months; ordinate: spermatozoa per $1 \mathrm{~mm}$ of ejaculate $\times 10^{6}$. Group 4-index 1; Group 5 -index 2 u 3 for dogs irradiated 6 and 3 years; index 4-control group

As a result, the reproductive ability of the male dogs in groups 3-5 decreased noticeably. The percentage of pregnant female dogs and the number of pups decreased progressively, while the number of stillborn pups increased. By the end of the third year of the exposure, the group 5 animals had shown the total male sterility. At the same time, the histomorphologic examination of the dog testicles demonstrated a relatively high level of stem cell preservation (the type A spermatoblasts amounted to 6-7\% of the reference level). The concentration of the spermatozoa began recovering in three months after stopping the 6-year exposure and reached about $30 \%$ of its normal level in seven months.

Investigation of male dogs descendants was made during 3 years after the end of irradiation. It was shown that the descendants reflected the regularities of the disorders revealed in fathers' spermatogenesis. The changes were mainly noted during embryogenesis and manifested by the sterility, mortality and the decreased number of puppies in the posterity.

\subsection{Histological Investigations of Organs and Tissues}

Among the structures of central nervous system, the cerebral cortex and hypothalamus have exhibited the most pronounced changes. The neuron hyperchromatism and the contraction of single nerve cells were often accompanied by the expressed melting of the chromatophilic substance and by the occurrences of single destroyed cells. The changes in the frontal and occipital lobes of cerebral cortex and in the hypothalamus proved to be more expressed in the hypothalamus by the dogs exposed to the higher dose loads (groups 3-5) and to rise with time. The necrosis of separate nerve cells was also observed.

In the system of hypothalamus - hypophysis gland - cortex of renal glands and in thyroid gland on the first year of exposure increase of the activity blood glands and their hyperplasia was observed. However to the end second year and through 3-th year exposure activity blood gland was hardly decreased. By the end of the second year and during the third year of the exposure of animals of groups 3-5, that process was replaced by depletion and decay of the functional activity of the glands. It was leaded to distress of blood gland to the end 3 -th year exposure. The hypothalamus disorders induced by the chronic exposure showed themselves as a fall of neurosecretion activity that was expressed to be a decreased content of the hypophysis hormones.

In different zones cortex of renal gland a dystrophic changes cells and necrotic sites were observed. In thyroid gland to the end second year exposure to 240-380 cSv doses hormonal activity was decreased also. These changes result to a decrease of the adaptive response of organism and in a weaker reaction of its hypophysis-adrenal system to external loads. As the accumulative doses increased, the occurrence frequency of the dystrophic changes increased also and they took the form of sclerosis of stroma and vessels. The said character of the changes relevant to the secretion activity and the structural disturbances in hypophysis, adrenal glands, thyroid gland, and sex glands is a reflection of the nonspecific reaction of organism to chronic stress and agrees completely with H. Selye's conception about the development of the general adaptation syndrome.

After three years of the exposure, the histological examinations of liver revealed the morphological changes that showed themselves as an enhancement variability of the dimensions of nuclei of the hepatocytes and as an increased number of the cells with double nucleus. Histological investigation of the hepatocytes in the animals of groups 4 and 5 showed the numerous disturbances, whose abundance and intensity increased with total equivalent dose (multiple small vacuoles appeared, which failed to react positively to lipids). Sclerotic changes in vessels, periportal tissue, and central veins were also in progress.

The morphological examinations of kidneys have shown that the vascular portion of nephron was the most injured. The walls of small and middle kidney arteries had nubs and was observed their constriction. The sclerosal glomerules occurred more frequently in the animals of groups 4 and 5 and were progressing in time. Thickening of the basic capillary membranes, denudation of capillary membranes, and sclerosis of glomerules were further observed. Enhancement of vascular disorders and development of sclerotic changes were also observed in the lung and heart vessels.

\subsection{Reaction of the Dog Organisms to Additional Functional Loads}

Use of additional functional loads has made it possible to find some disorders in the regulation processes and the 
insufficiency compensatory possibilities of the organism of the irradiated dogs. Starting from the second year of the exposure, the dogs of groups 3-5 showed a progressive deterioration of their cardiovascular system reaction to exercise stress, as compared with the control group. Investigators observed increase of the respiration frequency and obvious deviation of electrocardiography (ECG) date and a more long recovery time in irradiated dogs as compared with dogs of control group. Starting from the 20-th month of the exposure, the dogs of 3-6 groups sustained exercise stress poorly. After three years of the exposure, the percentage of such dogs reached $40 \%$. Some of them $(15 \%)$ refused to run at all. At the same time, all the dogs of the control group sustained exercise stress readily during all six years of the experiment. The reaction of the irradiated dogs to running included more high increase temperature of their bodies and skin and resulted to a much-increased intensity of the heat production and emission processes. In some cases, however, the balance of these processes was violated, with the changes showing different trends compared with the reference group. This means that the irradiated animals showed signs of abnormal thermoregulation, which was also confirmed by the fact that the irradiated dogs of 3-5 groups sustained the heat load worse compared with the control group.

Some of the irradiated dogs were excluded from the heat load experiment after they showed a dangerous aggravation of symptoms (a rapid decrease in their respiratory rate combined with an abrupt rise of their body temperature). All the above indicate the very serious disturbances of the thermoregulation processes in the irradiated dogs.

So, above considered a histological date and change reaction of organism by application of additional functional load were deposed about considerable reduction of the compensation reserves of organism. In the systems involved in regulating the physiological functions of an organism and in realizing its compensation-adaptation reactions develop with increase time of the exposure and equivalent doses the histomorphological changes that result to a decrease of the functional potential of these systems. The cases of insufficient compensatory reactions and reduction adaptability of organism were also observed in the dogs.

\subsection{Survivability and Life Span of the Dogs}

The late effects relevant to untimely deaths of the exposed animals were studied using 31 laboratory dogs irradiated for six years and 27 dogs irradiated for three years. Control group included 13 dogs. It should be noted that the early deaths of the dogs in groups 1-3 and in the control group were not caused by development of tumors in the animals, but were due mainly to the development of inflammatory processes, the aging-induced decrease of the dog organism resistibility to development of infectious processes. The principal causes of the deaths were most frequently found to be pneumonia and gastrointestinal tract disorder in the dogs. The development of malignant neoplasms was observed during a later period after the exposure. It should be noted that leucosis has never been diagnosed in the experimental dogs.
In the above experiment, the dogs of groups 1-5 developed carcinoma of the liver, urinary bladder, prostate gland, and submandibular glands, as well as malignant adenoma of the hypophysis and melanoma of the eye. The tumorogenesis in the exposed animals was characterized by the development of primary multiple tumors.

It is of a great practical interest to analyze the survivability curves for dogs exposed chronically to different dose rates (groups 1-3) and to a combined chronic and acute irradiation that simulated the impact of the deterministic radiation sources (galactic cosmic rays (GCR)) and of solar flares (groups 4 and 5) during a space flight. Figure 6 presents the survivability curves for the dogs of the experimental groups exposed during three and six years and for the control group dogs versus their age.

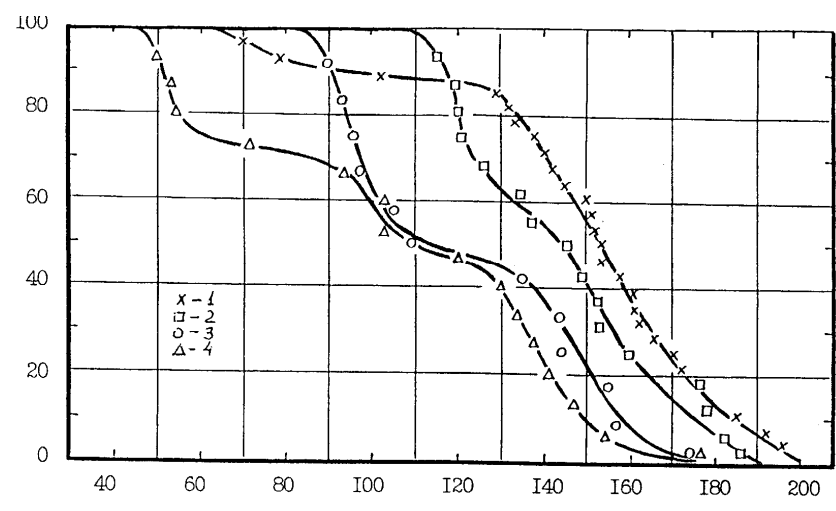

Figure 6. Survival of the male dogs exposed to acute and chronic irradiation on from age, \%

1 - date dogs of 1 and 2 groups, exposed with a dose $0-186 \mathrm{cSv}$

2 - date dogs of 2, 3 and 4 groups, exposed with a dose $360-375 \mathrm{cSv}$

3 - date dogs of 3 and 4 groups, exposed with a dose $720-750 \mathrm{cSv}$

4 - date dogs of 5 group, exposed with a dose 564-1128 cSv

Abscissa: age of dogs, months; ordinate survivability, \%

At least two animal death peaks can be noted, corresponding to earlier and later deaths. Having accumulated the doses of 63-186 cSv, the chronically irradiated dogs of groups 1-3 showed the mean life span that differed in no way reliably from that of the control dogs. Since the number of dogs in the irradiated experimental groups and control group is small (up to 13 animals), the dog groups that received similar doses were joined together to permit a quantitative analysis of the animal life span reduction on the absorbed dose level. The data displayed in Fig. 6 indicate that the dogs exposed to a somewhat higher dose rate (groups 2-5) died much earlier.

The analysis has shown that the 6-year chronic exposure dogs of group 2 with a dose rate $62 \mathrm{cSv} /$ year and the 3-year exposure of dogs groups 3 and 4 to a dose 360-372 cSv resulted in a $\sim 5 \%$ life span reduction. The 6-year exposure dogs of groups 3 and 4 to a dose $720-750 \mathrm{cSv}$ resulted in a much more significant reduction of the dog life span. As inferred from the nearest death peak, the life span got reduced by more than three years (by $\sim 30 \%$ ). The life span of the relatively stable animals (the second death peak at an age of 130-150 months) got reduced by about two years (by 17\%). 
The dogs of group 5 showed the most significant reduction of their life span. After 3 years of exposure to a dose $564 \mathrm{cSv}$ (including 9 acute fractioned exposure to a dose $42 \mathrm{cSv}$ per a fraction), the animal life span got reduced by 3-4 years (25$30 \%)$, as inferred from the data of the first and second death peaks. After 6-year exposure to a dose $1128 \mathrm{cSv}$, the life span estimated for less viable animals (the first death peak) decrease by factor 2.5 and approach to $40 \%$ of the life span of the control group dogs [2,8].

Analyzing the results of the actual long-term experiment has shown that the chronic irradiation of the dogs of groups 2 and 3 and, particularly, the combined chronic and-acute irradiation of the animals of groups 4 and 5 conduced during the exposure and later on, to a significant stress of the organism regulation system, to a higher exhaustion rate of the compensatory reserves of organism, to a speeded-up ageing, and to a fall of the resistance of organism to the additional functional load. As a result, the sickness rate and mortality of the experimental animals increased. A broad range of longterm adverse manifestations have been shown to arise in the animal organisms exposed to doses of 360-370 cSv (just the dose level permitted of the different cosmic agencies by the norms for career limits that received comparatively recently $[2,12,13]$. The frequency of these manifestation abrupt increased with dose exposure. The morphological changes in the organs and tissues (structures of central nervous system, endocrine glands of the irradiated animals, kidneys, liver, and vessel endothelium) of the irradiated animals are the nonspecific manifestations in response to long term external irradiation. They testify also about more quick and early development of age-related changes.

The reduction of the compensatory reserves founded in the experiments with chronic irradiation small and big laboratory animals has made it also possible to construct the mathematical model of the radiation-induced mammals death rate [2, 3, 14]. It predicts a parallel shift of the curves describing the dependence of the logarithm of the mortality rate on the age, after acute exposure from the different doses. The model also successfully predicts an increase in slope of the curves after chronic exposure to radiation at various dose rates. With used this model can satisfactorily describe the experimental data on the variations of the animals death rates after acute exposures to different doses and during chronic irradiations with different dose rates. As applied to human exposure, the model has made it possible to evaluate the total radiation risk for life expectancy of cosmonauts and to estimate a possible reduction of their life span.

\section{Radiation Risk in Flight}

Galactic cosmic rays are regarded as the main deterministic radiation hazard source in some of the possible Martian mission scenarios. Besides, the cosmonauts will suffer short-term stochastic impacts of solar cosmic rays (SCR). As mentioned above, the radiation risk of cosmonauts during an interplanetary space mission and the total radiation risk for their life expectancy can be evaluated in terms of the model for radiation-induced mammal death rate and using the generalized dose, which is the dosimetric criterion permitting the complicated character of irradiation in the space to be reduced to the standard radiation impact [1-3]. Mean-tissue equivalent dose rates in a spherical phantom for different spacecraft shielding thicknesses were taken from work [15] to calculate generalized dose values. The expressions for the mean-tissue equivalent dose rate (cSv/day) are:

$\mathrm{D}=1 / 365[5 \exp (-\mathrm{X} / 6.45)+24 \exp (-\mathrm{X} / 85.5)]$ at maximum SA
$\mathrm{D}=1 / 365[41.5 \exp (-\mathrm{X} / 2.8)+48 \exp (-\mathrm{X} / 85.5)]$ at minimum SA

where $\mathrm{X}$ is the $\mathrm{Al}$ shielding thickness, $\mathrm{g} / \mathrm{cm}^{2}$.

Galactic cosmic rays are characterized by a complicated composition and by a relatively hard spectrum that extends up to hundreds of $\mathrm{GeV}$. As follows from the above equation (3), the galactic radiation attenuates little as the shielding thickness increases. The annual doses during minimum of solar activity (SA) prove to be rather high and are 2-2.5 times as high as during maximum SA. Therefore, the actual Martian mission is planned to realize during phase of maximum solar activity, so that the physical shielding weight for the Martian spacecraft would be much reduced and determined mainly by life support systems.

The solar energetic proton (SEP) events are of short-term nature and the solar cosmic ray spectrum is softer compared with galactic cosmic rays. This means that a space-oriented radiation shelter can well provide against SEP impact. The temporal SEP event evolution is a stochastic process as regards the total flux and energy spectrum of protons. The results of analyzing the SEP events of cycles 19 and 20 have underlain the State Standard "Solar Cosmic Rays. Model of the Proton Flux" [16]. The standardized model was used to calculate the equivalent dose induced by solar cosmic rays in a cosmonaut body during a Martian mission and to evaluate the generalized dose with a view of calculating the radiation risk. Equivalent and generalized dose values for different shielding thicknesses and in various marrow locations of an anthropomorphic phantom [17] have been calculated in works [2, 3].

The generalized doses for a long-term interplanetary space flight were calculated in terms of the Monte-Carlo statistical test method by sampling $10^{4}$ flight histories at random. In each of the histories, the calculated generalized doses induced by galactic cosmic rays were added to the generalized doses from the short-term solar cosmic ray impacts that were also sampled at random in terms of the standardized proton flux model [16].

On Fig.7. are showed values demographic risk for man in Russia and mean values of radiation risk are calculated for cosmonauts of 40 year age dependent on the duration of flight (months) beyond Earth's magnetosphere in phase maximum SA for different shelter shielding thicknesses $\left(X_{\text {sst }}\right.$ $\left.\mathrm{g} / \mathrm{cm}^{2} \mathrm{Al}\right)$. We was assumed, that habitat shielding thicknesses $X_{\mathrm{hmst}}=1 \mathrm{~g} / \mathrm{cm}^{2}$. 


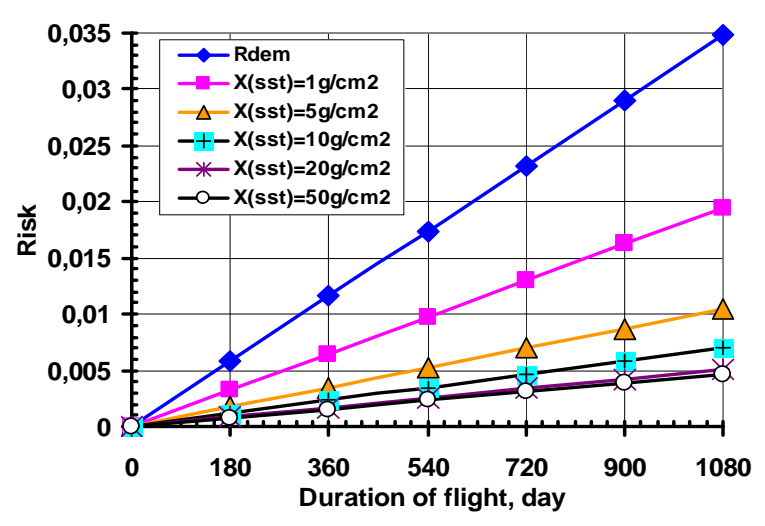

Figure 7. Demografic risk for man in Russia and radiation risk in flight for cosmonauts 40 year age dependent on the duration of flight (months) beyond Earth's magnetosphere in phase maximum SA for different shelter shielding thicknesses $\left(\mathrm{X} \mathrm{g} / \mathrm{cm}^{2} \mathrm{Al}\right)$.

As spacecraft shielding thickness increases this radiation risk greatly reduces. It was shown for interplanetary flight conditions, at the real spacecraft shielding thickness (10-20 $\mathrm{g} / \mathrm{cm}^{2}$ ), that additional radiation risk during the flight may be as high as 10 to $20 \%$ of demographic risk for the same time duration (the demographic risk is the mortality rate for population at a given age). Calculations indicated, that radiation risk for a flight duration 1-2 year in the period maximum of SA consist in range $\left.(1,7 \div 4,4) 10^{-3}\right)$. Further increase spacecraft shielding thickness is none effective $[2,3]$.

\section{Life Total Radiation Risk for Cosmonauts after Mars Mission}

The reduction of the compensatory reserves founded in the experiments with chronic irradiation small and big laboratory animals has made it also possible to construct the mathematical model of the radiation-induced mammals death rate. Total radiation risk for their life expectancy can be evaluated in terms of the model for radiation-induced mammal death rate and using the generalized dose, which is

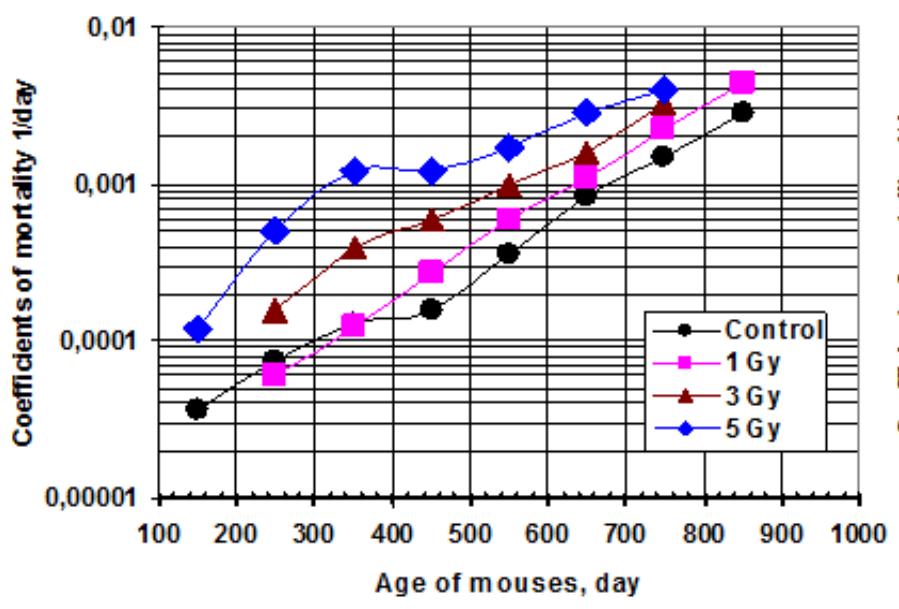

A the dosimetric criterion permitting the complicated character of irradiation in the space to be reduced to the standard radiation impact $[2,3,14]$. The values total volume of compensatory reserves organism $\mathrm{Q}(\mathrm{t})$ and increase mortality rate mammals $\mu^{\text {rad }}(t)$ proceed on exponential law with certain age. Velocity these processes intensify in result radiation exposure with different dose rate and total equivalent dose or generalized dose $\mathrm{H}_{\mathrm{L}}$ :

$$
\begin{aligned}
& \quad \mathrm{Q}(\mathrm{t})=\mathrm{Q}_{0} \exp \left[-\lambda_{0}-\mathrm{B} \gamma^{\delta}\right]\left(\mathrm{t}-\mathrm{t}_{0}\right) \approx \\
& \approx \mathrm{Q}_{0} \exp \left[-\lambda_{0}\left(\mathrm{t}-\mathrm{t}_{0}\right)-\mathrm{B} \mathrm{H}_{\mathrm{L}}\right] ; \\
& \mu_{\mathrm{rad}}(\mathrm{t})=\mu\left(\mathrm{t}_{0}\right) \exp \left[\left(\lambda_{0}+\mathrm{B} \gamma^{\delta}\right]\left(\mathrm{t}-\mathrm{t}_{0}\right) \approx\right. \\
& \approx \mu\left(\mathrm{t}_{0}\right) \exp \left[\lambda_{0}\left(\mathrm{t}-\mathrm{t}_{0}\right)+\mathrm{B} \mathrm{H}_{\mathrm{L}}\right]
\end{aligned}
$$

Model predicts a parallel shift of the curves describing the dependence of the logarithm of the mortality rate on the age, after acute exposure from the different doses. The model also successfully predicts an increase in slope of the curves after chronic exposure to radiation at various dose rates. With used this model can satisfactorily describe the experimental data on the variations of the animals death rates after acute exposures to different doses and during chronic irradiations with different dose rates. On Fig.8. was showed facility of model for description some experimental dates after acute exposure from the different doses and after chronic exposure to radiation at various dose rates. On basis experimental data was received evaluated significance coefficient $B(B=0,091 / \mathrm{Sv})$.

As applied to human exposure, the model has made it possible to evaluate the total radiation risk for life expectancy of cosmonauts and to estimate a possible reduction of their life span. The total radiation risk (TRR) throughout the cosmonaut life expectancy, including the death risk of neoplasms and possible development of long-term radiation damages in different organism systems, was calculated with used of the model for radiation-induced mammals death rate, which determines the obligate variations of the compensatory reserves of organism due to radiation impact $[2,3,18]$.

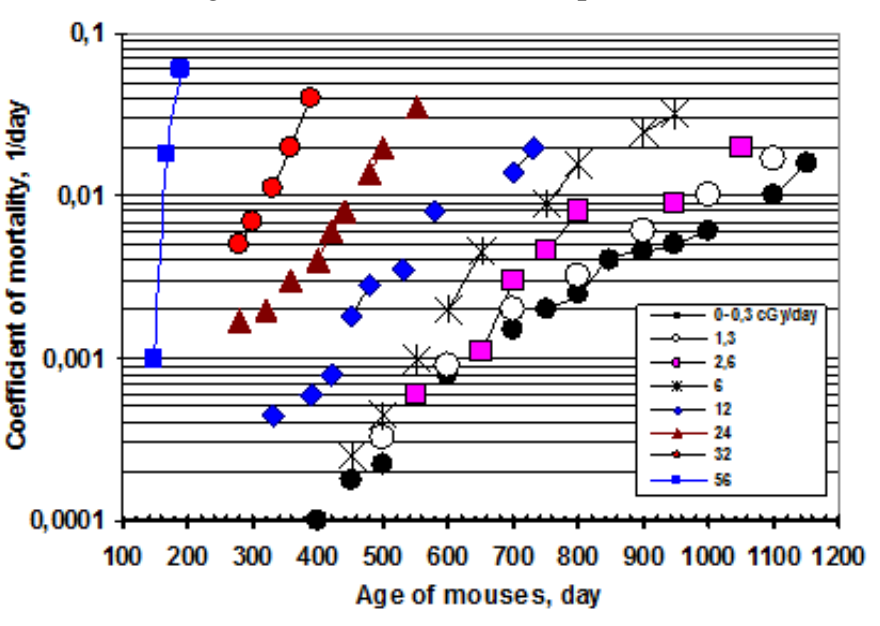

B

Figure 8. Dependence mortality rate mouse from age after acute exposure in different doses, Gy (A) and after chronic exposure mouse rates cGy/day (B) 
On the basis of the equations, inferred from the model of the radiation-induced rate mortality of mammals, and estimates of generalized dose $\mathrm{H}_{\mathrm{L}}$ it is possible to predict additional radiation mortality risk for any period of cosmonauts' lifetime, as well as the possible shortening of life expectancy. Mean values of radiation risk was calculated for cosmonauts of various ages dependent on the duration of flight beyond Earth's magnetosphere, spacecraft shielding thickness and different phases of SA.

Previous it was shown for interplanetary flight conditions, at the real spacecraft shielding thickness $\left(10-20 \mathrm{~g} / \mathrm{cm}^{2} \mathrm{Al}\right)$, that radiation risk during the flight may be as high as 10 to $20 \%$ of demographic risk for the same time duration (the demographic risk is the mortality rate for population at a given age). As spacecraft shielding thickness increases this radiation risk greatly reduces and in the period maximum of SA consist in range $(1,7 \div 4,4) 10^{-3}$ for a flight duration $1-2$ year. Calculations of total radiation risk over the entire lifetime for cosmonauts which was included the mortality up to 70 years age caused by radiation exposure from tumor and all other death causes have shown that it does not depend on

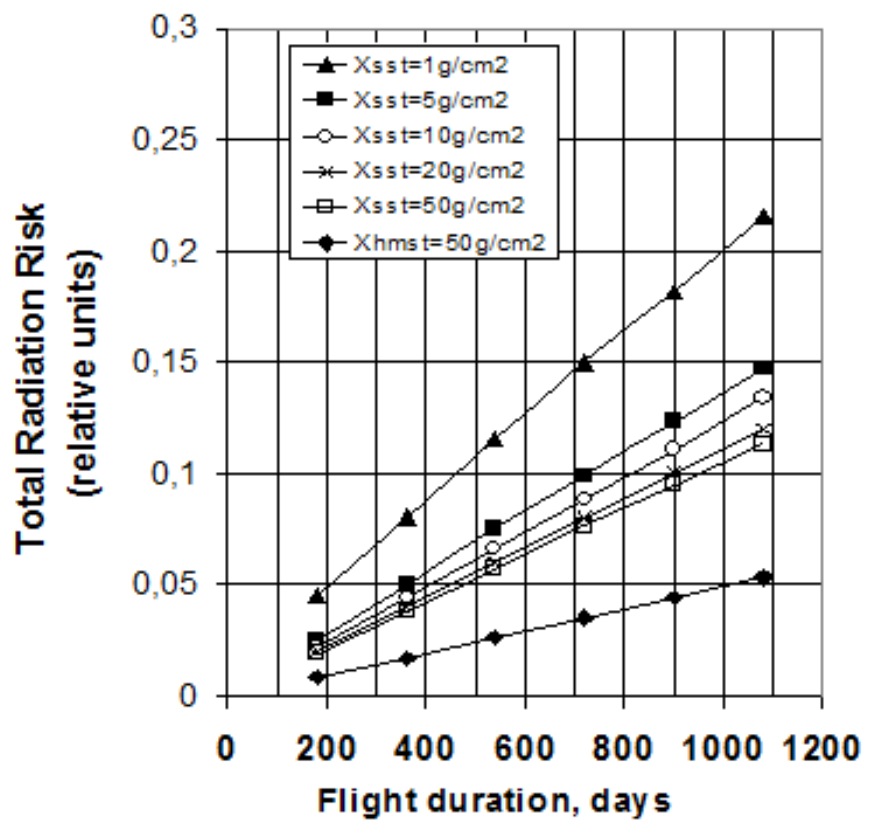

A the age and is 20-30 times as high as that during flight [2, 3, $18]$.

On Fig. 9 was presented dependencies of TRR over the lifetime for cosmonauts aged 40 years (A) and their life shortening LS (B) on interplanetary flight duration in the phase of maximum SA for various spacecraft shielding thicknesses.

It should be mentioned that as the radiation shelter shielding thickness (Xsst) increases in the range from 1 to 20 $\mathrm{g} / \mathrm{cm} 2$, TRR and LS values are significantly reduced. Further increase in the radiation shelter shielding thickness Xsst and minimum habitat module shielding thickness $(\mathrm{Xhmst}=1$ $\mathrm{g} / \mathrm{cm}^{2}$ ) does not change these values, because of the predominating contribution of GCR in the total generalized dose. In case of increasing the habitat module shielding thickness up to $50 \mathrm{~g} / \mathrm{cm}^{2}$ and absence of the special radiation shelter the radiation risk value over the lifetime is reduced (more than 4 times compared to that for minimum habitat module shielding thickness), however it results in a significant increase in the weight of the spacecraft shielding.

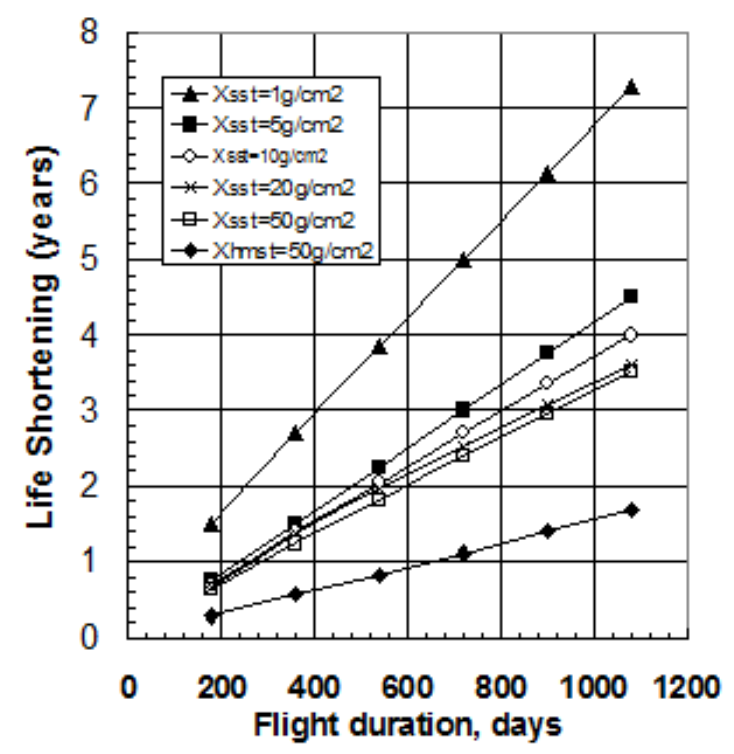

$\mathrm{B}$

Figure 9. Dependences of TRR over the lifetime for cosmonauts aged 40 years (relative units A) and their life shortening (LS, year - B) on interplanetary flight duration in the phase of maximum SA for various spacecraft shielding thicknesses (Xsst, g/cm2) and habitat shielding thicknesses (Xhmst, g/cm2).

Figure 10 illustrates comparative estimates of TRR and cancer risks over the lifetimes of cosmonauts dependent on the age of the cosmonauts after 2 year-long interplanetary flight in the phase maximum SA for habitat module shielding thickness $(X h m s t=30 \mathrm{~g} / \mathrm{cm} 2)$. To maximum and minimum assessments of cancer risks we used data works [2, 3, 13].

From date of the Fig.10 it is obvious that cancer risk strongly depends on the age of the cosmonauts, while TRR over the lifetime of the cosmonauts exceeds the maximum estimate of cancer risk and is independent of age. As it can be seen, for 25-year-old cosmonauts TRR and cancer risk are similar. At the same time for 50-year-old cosmonauts TRR is more than 3 times as high as the cancer risk over a lifetime. Therefore TRR for senior-age groups is related more to nontumor death causes. In particular, a significant part of TRR can result from vascular changes and blood-circulation abnormalities after irradiation at levels below the career limit, established in USA (NCRP (1989) [13] and in USSR Methodical instructions MI 2.6.1. 44-03-2004. USSR [19]. 


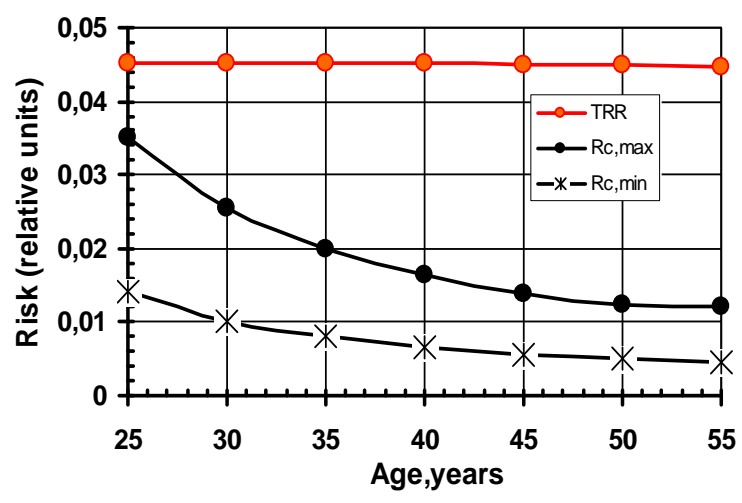

Figure 10. Dependences of TRR and maximum and minimum assessments of cancer risk over lifetimes of cosmonauts (relative units) on their age after 2 year-long interplanetary flight in the phase maximum SA for habitat module shielding thickness (Xhmst $=30 \mathrm{~g} / \mathrm{cm} 2)$.

Studies of the survived peoples after use nuclear bomb in Japan and epidemiological data show an increase in noncancer mortality with radiation dose. There are deaths from cardiovascular and neurovascular diseases, blood-circulation abnormalities and insult of brain [20,21]. This is confirmed by clinical observations of the occupationally exposed workers on Earth. These diseases in radiation workers are larger then for other fields of work [20-22].

Calculation of total radiation risk over the entire lifetime for cosmonauts at generalized dose $1 \mathrm{~Sv}$ have shown that it amount $13 \%$. Cancer risk at generalized dose $1 \mathrm{~Sv}$ for cosmonauts and astronauts in age 40 years amount about $3 \%$, what in 3 - 4 time below TRR [2, 3]

Epidemiological data in series works investigators of Russia, European countries, USA [22-24] was showed also what TRR on $1 \mathrm{~Sv}$ achieve 10-14\% and exceed cancer risk over a lifetime in 3 once.

\section{Conclusion}

It can be concluded that the total radiation risk for cosmonauts after flights is approximately 30 times as high as the in-flight radiation risk and several times as high as the cancer risk occurring over their lifetimes. Since the total radiation risk parameter does not depend on the in-flight age of cosmonauts, it makes this parameter preferable than the cancer risk to normalize radiation impact and to establish radiation dose limits for cosmonauts over their careers. Along with this parameter, estimates of possible shortening of lifetime can also be obtained.

\section{References}

[1] Shafirkin A.V. and Petrov V.M., "Estimation of a cosmonaut's radiation hazard during long-term space mission on the basic of a generalized dosimetric function," Adv. Space Res., vol. 30, No. 4, 2002, pp. 995-998

[2] Shafirkin A.V., Grigoriev Yu.G., "Interplanetary and orbital space flight. The radiation risk to astronauts. Radiobiological Basis. Economica” 2009, 639 p.
[3] Grigoriev Yu.G., Ushakov I.B., Krasavin E.A., Davidov B.I., Shafirkin A.V., "Space radiobiology for 55 years (To 50-years State Research Center Institute of biomedical problems Russian Academy of Sciences). Economica,” 2013, 301 p. (in Russian)

[4] Shafirkin, A.V. and Fedorenko B. S. "Substantiation of the Dependence of Radiation Quality Coefficients on LET in Application to the Assesment of Early Radiobiological Effects," Aviakosmicheskaya and ecologicheskaya meditsina, vol. 32, No. 2, 1998. pp. 4-9. (in Russian)

[5] GOST 25645.218-90. "Space Crew Radiation Safety during Space Flight. Dependence of Cosmic Radiation Quality Factor from Linear Energy. Gosstandart USSR,” 1991, Moscow. (in Russian)

[6] Blair H.A., "Some aspects properties of reparable and irreparable radiation injury. Some aspects of internation irradiation," Oxford: Pergamon Press, 1962, pp. 147-150.

[7] Blair H.A., "The constancy of repair rate and irreparability during protracted exposure to ionizing radiation," Ann. New York Acad. Sci., vol. 114, part.1, 1964, pp.150-157.

[8] Grigorjev, Yu. G., Popov V.I., Shafirkin A.V., Antipenko J.B., "Somatic Effects of Chronic Gamma-Irradiation," Moscow, Energoatomizdat, 1986. 196 p. (in Russian)

[9] GOST 25645.219-90., "Space Crew Radiation Safety during Space Flight. The Model Takes into Consideration the Influence of Spatial Nonuniformity Irradiation on the Generalized Radiobiological Effect. Gosstandart USSR," Moscow, 1991. (in Russian)

[10] Saksonov, P.P., Antipov V.V., Davidov B. I., "The Essay of Space Radiobiology, In.: The Problems of Space Biology,” vol. IX, Moscow, "Science”, 1968, 532 p. (in Russian)

[11] Antipov, V.V., Davidov B. I., Verigo V.V., Svirijev Yu. M., "About the Combined Impact of Different Factors in Space Flight. In.: Foundations of Space Biology and Medicine," Moscow, "Science", vol. 2, book 2, 1975, pp. 243-267. (in Russian)

[12] GOST 25645.215-85. 1986. Space Crew Radiation Safety during Space Flight. Safety norm for flight durations up to three years, Gosstandart USSR, Moscow

[13] NCRP. Report 98, "Guidance on Radiation Received in Space Activities National Council on Radiation Protection and Measurement," Bethesda, MD, 1989.

[14] Shafirkin, A.V., "The Model of the Rate of Radiation-Induced Mammalian Death Based on Determination of Delayed Consequences of Different Doses of Radiation," Aviakosmicheskaya and Ecologicheskaya Meditsina, vol. 33, No. 4, 1999, pp. 64-69. (in Russian)

[15] Dudkin V.E., Potapov Yu.V., "Doses from galactic cosmic ray particles under spacecraft shielding," Nucl. Tracks Radiat. Meas., vol. 20, No. 1, 1992, pp. 33-39.

[16] GOST 25645. 134-86, "Space Crew Radiation Safety during Space Flight. The Solar Cosmic Rays. Model of the Proton Flux.," Gosstandart USSR, Moscow, 1986. (in Russian)

[17] GOST 25645.203-83, "Space Crew Radiation Safety during Space Flight. The Model of Body for Calculations of Tissue Doses," Gosstandart USSR, Moscow, 1984. (in Russian) 
[18] Shafirkin A.V., Petrov V.M., Kolomensky A.V., Shurshakov V.A., "Lifetime total radiation risk of cosmonauts for orbital and interplanetary flights," Adv. Space Res., vol. 30, No. 4, 2002, pp. 999-1003.

[19] Methodical instructions MI 2.6.1. 44-03-2004 USSR, Limitation irradiation of cosmonauts at orbital cosmic flights. Federal Cosmic Agency, Moscow, 2004

[20] Robertson, T.L., Shimizu Y., Kato H., et al., "Incidence of stroke and coronary heart disease in atomic bomb survivors, living in Hiroshima and Nagasaki 1954-1974," RERFTR, 1979 , pp. 12-79.

[21] Kadama, K., Shimizu Y., Sawada H., et al. "Incidence of stroke and coronary heart disease in adult study sample 19581978,” RERFTR, 1984, pp. 22-84.

[22] Azizova T.V., "The Nervous System Condition of Persons
Undergoing to Chronic Professional Exposure by Ionizing Radiation (35-45 Year Period of Observations)," Dissertation State Research Center - Institute of Biophysics by Ministry of Health R.F., Moscow, 1999. (in Russian)

[23] Geard C.R., Jenkins-Baker G., Grabham P. et al., "Human endothelial cells in $2 \mathrm{D}$ and 3-D system. Noncancer effects and space-related radiations," 4-th Internation Workshop on Space Radiation Research and 17-th Annual NASA Space Radiation Health Investigators Workshop, Dubna, 2006, pp. 34-35.

[24] Little M.P., Azizova T.V.,Bazika D., Bouffler S.D., Cardis E. et.al., "Systematic Review and Meta-analysis of Circulatory Disease from Exposure to Low-Level Ionizing Radiation and Estimates of Potential Population Mortality Risks," Environmental Health Perspectives, vol. 120, No. 11, 2012, pp. 1503-1511 